\title{
AUTOMORPHIC GROUP REPRESENTATIONS: \\ A NEW PROOF OF BLATTNER'S THEOREM
}

\author{
P. DE LA HARPE AND R. J. PLYMEN
}

\section{Introduction}

There are many constructions which give rise to the hyperfinite $\mathrm{II}_{1}$ factor $\mathfrak{A}$ of Murray and von Neumann [12]. For example:

(i) the infinite tensor product of a countable number of matrix algebras with respect to their traces;

(ii) the group measure space construction from an ergodic measure preserving transformation;

(iii) the left regular representation of a locally finite discrete group with infinite conjugacy classes;

(iv) the von Neumann algebra generated by the CAR algebra of an infinitedimensional Euclidean space $E$ in its trace representation.

To each of these ways of obtaining $\mathfrak{A}$ correspond automorphisms of $\mathfrak{A}$. The construction that will interest us is the fourth one. The automorphisms in question arise naturally in the following way. The vectors in $E$ generate $\mathfrak{A}$ as a von Neumann algebra; the full orthogonal group $O(E)$ of $E$ acts on $E$, hence on $\mathfrak{2}$ as automorphisms.

The normal subgroup $G_{2}$ of $O(E)$ which acts as inner automorphisms of $\mathfrak{A}$ is of particular interest. The group $G_{2}$ was determined by Blattner [2]. The normal subgroup $G_{1}$ of $O(E)$ which acts as inner automorphisms of the CAR algebra is also of interest, and was determined by Shale and Stinespring [21]. The purpose of this article is to give new, simpler proofs of these two results.

We quote a result of the first-named author according to which each element of a proper normal subgroup of $O(E)$ is a compact perturbation either of the identity operator $I$ or of $-I$. We prove directly that $G_{1}$ and $G_{2}$ are proper normal subgroups of $O(E)$ and then exploit the spectral theory of compact operators. Although our proofs are new, they make liberal use of original ideas of Blattner, Shale and Stinespring. It is illuminating to prove the two basic results simultaneously. Our proofs are naive and constructive: corresponding to the spectral decomposition of an orthogonal operator in $G_{2}$, the implementing unitary operator in $\mathfrak{Y}$ is explicitly constructed as an infinite product.

Let $\mathscr{C}$ denote the CAR algebra. Since the centres of $\mathfrak{A}$ and $\mathscr{C}$ comprise scalar multiples of the identity, each implementing unitary operator is determined up to such a scalar. Consequently we have projective unitary representations of $G_{1}$ and $G_{2}$ as follows:

$$
G_{2} \rightarrow U(\mathfrak{Q}) / U(1), \quad G_{1} \rightarrow U(\mathscr{C}) / U(1)
$$

Received 1 June, 1978.

[J. London Math. Soc. (2), 19 (1979), 509-522] 
where $U(\mathfrak{R})$ and $U(\mathscr{C})$ denote the unitary groups in $\mathfrak{A}$ and $\mathscr{C}$ respectively. When $E$ is of finite dimension $2 n$, these representations become the spin representation

$$
O(E) \rightarrow U\left(2^{n}\right) / U(1) \text {. }
$$

This aspect, already noted by Shale and Stinespring, has been developed by the present authors in [9] and [13].

In Section 2 we recall various definitions and facts concerning orthogonal groups and the CAR algebra. In Section 3, the production of an outer automorphism of $\mathfrak{A}$, and the above result on proper normal subgroups of $O(E)$, leads readily to the following result: a separable locally compact group acts faithfully by outer automorphisms of $\mathfrak{A}$. This result is due to Blattner [2], and, for the special case of a discrete group, to Suzuki [24]; see also Choda [25], and Nakamura and Takeda [26]. Sections 4 and 5 are devoted to the proofs of the two basic results. We have tried to make these proofs as self-contained as possible. We avoid the use of unbounded operators.

The first-named author has benefited from conversations with V. Jones, and has been partly supported by the "Fonds national suisse pour la recherche scientifique".

\section{Various orthogonal groups and statement of the main results}

We first review basic facts on the Clifford algebra of a real Hilbert space: details can be found in any one of $[2,9,10,14,21]$. The classical theory of finite-dimensional Clifford algebras appears in Atiyah, Bott and Shapiro [1].

Let $E$ be a real Hilbert space, with inner product denoted by $\langle. \mid$.$\rangle . Let \mathrm{Cl}(E)$ be the Clifford algebra of the quadratic form $q$ on $E$ defined by $q(e)=\langle e \mid e\rangle=\|e\|^{2}$. We denote by $X \mapsto X^{*}$ both the main anti-automorphism of $\mathrm{Cl}(E)$, namely that which restricts to the identity on $E$, and its semi-linear extension to the complexified algebra $\mathrm{Cl}(E)^{\mathbb{C}}=\mathrm{Cl}(E) \otimes_{\mathbb{R}} \mathbb{C}$. Both $\mathrm{Cl}(E)$ and $\mathrm{Cl}(E)^{\mathbb{C}}$ are $\mathbb{Z}_{2}$-graded algebras. It is easy to check that $\mathrm{Cl}(E)^{\mathbb{C}}$ admits a unique normalized central even linear form $\tau$, which is called the trace. "Even" means that $\tau$ vanishes on the odd part of the grading, "central " means that $\tau(X Y)=\tau(Y X)$ and " normalized" that $\tau(1)=1$.

If $F$ is a subspace of $E$, there is a natural injection of $\mathrm{Cl}(F)^{\mathbb{C}}$ into $\mathrm{Cl}(E)^{\mathbb{C}}$; indeed, if $F$ is closed in $E$, then $\mathrm{Cl}(E)^{\mathbb{C}}$ is the graded tensor product of $\mathrm{Cl}(F)^{\mathbb{C}}$ and $\mathrm{Cl}\left(F^{\perp}\right)^{\mathbb{C}}$, where $F^{\perp}$ is the orthogonal complement to $F$. By uniqueness of the trace, the restriction of $\tau$ to $\mathrm{Cl}(F)^{\mathbb{C}}$ is the trace on $\mathrm{Cl}(F)^{\mathbb{C}}$.

When $F$ has dimension $2 n$, it is a standard fact that $\mathrm{Cl}(F)^{\mathbb{C}} \cong \mathbb{C}\left(2^{n}\right)$, the algebra of $2^{n} \times 2^{n}$ matrices over $\mathbb{C}$; see [1]. If $E$ is infinite dimensional, then both $\mathrm{Cl}(E)^{\mathbb{C}}$ and its even part are simple algebras; both the centres coincide with scalar multiples of the identity.

From now on, $E$ will always denote an infinite dimensional real Hilbert space. Note that, as we shall be interested in complexified algebras, we could equally as well have started with the negative definite quadratic form $e \mapsto-\|e\|^{2}$ [9].

A prehilbert space structure is defined on $\mathrm{Cl}(E)^{\mathbb{C}}$ by $\langle X \mid Y\rangle=\tau\left(X Y^{*}\right)$; we denote by $H_{0}$ the complex Hilbert space obtained by completion. We denote the continuous extension of $\tau$ to $H_{0}$ by the same letter, and we write $\|\cdot\|_{2}$ for the norm defined by the inner product on $H_{0}$; the natural inclusion of $E$ in $H_{0}$ is an isometry. The left multiplication by any $X$ in $\mathrm{Cl}(E)^{\mathbb{C}}$ extends to a bounded operator $\pi_{0}(X)$ on $H_{0}$; we denote its norm by $\|X\|_{\infty}$. The completion $\mathscr{C}(E)$ of $\mathrm{Cl}(E)^{\mathbb{C}}$ with respect to this norm is a uniformly hyperfinite $C^{*}$-algebra known as the $C^{*}$-Clifford algebra, 
or the CAR algebra over $E$. If 1 denotes again the unit in this algebra, the triple $\left(H_{0}, \pi_{0}, 1\right)$ can be identified with the cyclic representation of $\mathscr{C}(E)$ associated to $\tau$ by the Gelfand-Naimark-Segal construction. If $E$ were finite dimensional, then $\pi_{0}$ would be the left regular representation of $\mathrm{Cl}(E)^{\mathbb{C}}$.

The weak closure $\mathfrak{A}(E)$ of the image of $\pi_{0}$ in the algebra of bounded operators on $H_{0}$ is a von Neumann algebra; indeed, it is easy to check that $\mathfrak{A}(E)$ is the hyperfinite factor of type $\mathrm{II}_{1}$. We shall identify $\mathrm{Cl}(E)^{\mathbb{C}}$ and $\mathscr{C}(E)$ with subalgebras of $\mathfrak{A}(E)$, and the latter to a subspace (not closed) of $H_{0}$. The operator norm on $\mathfrak{A}(E)$ will again be denoted by $\|.\|_{\infty}$. Each algebra introduced so far is $\mathbb{Z}_{2}$-graded: $\mathscr{C}(E)=\mathscr{C}(E)^{+} \oplus \mathscr{C}(E)^{-}$, $\mathfrak{U}(E)=\mathfrak{U}(E)^{+} \oplus \mathfrak{A}(E)^{-}$.

The theorems we are concerned with set up a basic relationship between some groups of unitary operators in the $\mathrm{II}_{1}$ factor $\mathfrak{A}(E)$ on the one hand, and some groups of unitary operators in the "real factor of type $I_{\infty}$ " on the other hand. We now describe this relationship.

Let $O(E)$ be the group of orthogonal operators on $E$, also called canonical transformations. From the universal properties of $\mathrm{Cl}(E)$, it follows that any $R$ in $O(E)$ extends uniquely to an automorphism $\mathscr{C}(R)$ of $\mathscr{C}(E)$ and also to an automorphism $\mathfrak{A}(R)$ of $\mathfrak{A}(E)$; this defines homomorphisms form $O(E)$ to the groups of * automorphisms of $\mathscr{C}(E)$ and $\mathfrak{A}(E)$ respectively. The images consist of the so-called special automorphisms. Special automorphisms are called Bogoliubov automorphisms in mathematical physics. A * automorphism $\phi$ of $\mathscr{C}(E)$ (respectively of $\mathfrak{U}(E)$ ) is inner if there exists a unitary element $U$ in $\mathscr{C}(E)$ (respectively in $\mathfrak{A}(E)$ ) with $\phi=\operatorname{Ad}(U)$, namely with $\phi(X)=U X U^{*}$ for all $X$ in the algebra. The centres of both $\mathscr{C}(E)$ and $\mathfrak{U}(E)$ coincide with scalar multiples of the identity. If $\mathfrak{P}(R)=\operatorname{Ad} U$ with $U$ unitary in $\mathfrak{R}(E)$ then we have

$$
\operatorname{Ad} U=\mathfrak{2}((-I) R(-I))=\alpha(\operatorname{Ad} U) \alpha^{-1}=\operatorname{Ad} \alpha(U)
$$

where $\alpha=\mathfrak{A}(-I)$. Consequently $\alpha(U)=\lambda U$ with $\lambda$ a scalar of modulus 1 . But $\alpha$ has period 2 so $\lambda^{2}=1$. Therefore either $\alpha(U)=U$ or $\alpha(U)=-U$. When $\alpha(U)=U$, $U$ lies in $\mathfrak{A}(E)^{+}$and $\mathfrak{A}(R)$ is called even; when $\alpha(U)=-U, U$ lies in $\mathfrak{A}(E)^{-}$and $\mathfrak{A}(R)$ is called odd. Similar terminology applies to $\mathscr{C}(R)$.

Define $O^{+}(E)$ to be the set (not a group) of those $R$ in $O(E)$ for which the eigenspace $\{e \in E: R e=-e\}$ is either infinite-dimensional, or finite even-dimensional; let $O^{-}(E)$ be the complement of $O^{+}(E)$ in $O(E)$. It is shown in Putnam and Wintner [17] that $O^{+}(E)$ is the image by the exponential map of the space of skew-adjoint operators on $E$. Let us denote by $C_{1}(E)$ (respectively $C_{2}(E), C_{\infty}(E)$ ) the set of all nuclear (respectively Hilbert-Schmidt, compact) operators on $E$; each is clearly an ideal in the algebra of all bounded operators on $E$. If $p=1,2, \infty$, then the sets

$$
\begin{aligned}
S O(E)_{p} & =\left\{R \in O^{+}(E): R-1 \in C_{p}(E)\right\} \\
O(E)_{p} & =\left\{R \in O(E): R-1 \in C_{p}(E)\right\} \\
O^{t}(E)_{p} & =S O(E)_{p} \cup\left\{R \in O(E):-R \in O^{-}(E) \text { and } R+1 \in C_{p}(E)\right\} \\
O^{\max }(E)_{p} & =O(E)_{p} \cup\left\{R \in O(E): R+1 \in C_{p}(E)\right\}
\end{aligned}
$$

are all normal subgroups of $O(E)$ (the superscript $t$ means "twisted"). The lattice 
of subgroups is

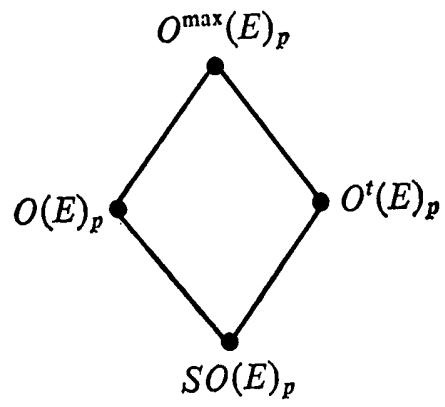

The quotient $O^{\max }(E)_{p} / S O(E)_{p}$ is $\mathbb{Z}_{2} \times \mathbb{Z}_{2}$. Quite specifically, let us denote by $O^{b}(E)_{p}$ the complement of $S O(E)_{p}$ in $O^{t}(E)_{p}$; then the complement of $S O(E)_{p}$ in $O(E)_{p}$ is $\left\{R \in O(E):-R \in O^{b}(E)_{p}\right\}$ and the complement of $O(E)_{p} \cup O^{t}(E)_{p}$ in $O^{\max }(E)_{p}$ is $\left\{R \in O(E):-R \in S O(E)_{p}\right\}$. Incidentally, $O(E)_{1}$ is denoted by $O\left(E ; C_{1}\right)$ in [9] and $O(E)_{2}$ is defined in [15]. Also, $S O(E)_{p}$ is a subgroup of index two in $O(E)_{p}$ and $O^{t}(E)_{p}$; each of these is, in turn, a subgroup of index two in $O^{\max }(E)_{p}$.

If $R$ lies in $O^{\max }(E)_{\infty}$, then $E \otimes_{\mathbb{R}} \mathbb{C}$ has an orthonormal basis of eigenvectors of $R \otimes 1$. This property is crucial in the following discussion.

We pause here for a short digression. The group $O(E)_{1}$ has a standard bi-invariant metric which assigns to two operators the nuclear norm of their difference. Using the same method as that used in Putnam and Wintner [17] one shows that the connected component of the topological group defined in this way is precisely $S O(E)_{1}$. It follows that $O^{t}(E)_{1}$ (respectively $\left.O^{\max }(E)_{1}\right)$ can be looked at as a topological group having two (respectively four) connected components, with $S O(E)_{1}$ again as the component of the identity. This remark carries over to the cases $p=2$ and $p=\infty$ (and indeed to any $p$ with $1 \leqslant p \leqslant \infty$ for the appropriate definitions in terms of the von Neumann-Schatten classes of operators $\left.C_{p}(E)\right)$. It is also true that the natural injection $O^{\max }(E)_{1} \rightarrow O^{\max }(E)_{2}$ is continuous with dense range, as is the natural injection $O^{\max }(E)_{2} \rightarrow O^{\max }(E)_{\infty}$. This ends the digression.

The main results are the following:

Theorem 1 (Shale and Stinespring). Let $R \in O(E)$. Then

(i) $\mathscr{C}(R)$ is inner if and only if $R \in O^{t}(E)_{1}$

(ii) $\mathscr{C}(R)$ is inner and even if and only if $R \in S O(E)_{1}$.

Theorem 2 (Blattner). Let $R \in O(E)$. Then

(i) $\mathfrak{P}(R)$ is inner if and only if $R \in O^{t}(E)_{2}$

(ii) $\mathfrak{Q}(R)$ is inner and even if and only if $R \in S O(E)_{2}$.

In the original papers, Blattner's theorem came first [2]. Shale and Stinespring obtained their theorem as a corollary of a result which is itself close to Theorem 2 (see [20; Section 3]). In Sections 4 and 5 below, we shall prove the theorems independently of each other; we shall express the arguments simultaneously: most lemmas will have statements (i) and (ii).

The notations $\mathscr{C}(R), \mathscr{C}(E), \mathfrak{U}(R), \mathfrak{A}(E)$ have been used because $\mathscr{C}$ and $\mathfrak{Y}$ are functors. This aspect has been extensively developed by Schrader and Uhlenbrock n [19]. 


\section{Special automorphic group representations}

Let Aut $(\mathfrak{H})$ denote the group of automorphisms of $\mathfrak{A}(E)$ as a $*$ algebra. Then Aut $(\mathfrak{Q})$ is the group of automorphisms of $\mathfrak{Q}(E)$ as a von Neumann algebra, because the ultraweak topology on $\mathfrak{A}(E)$ is determined by the normal states and these are determined by the order structure which is preserved by automorphisms (see [6; Chapter I, §4]). As $E$ generates $\mathfrak{A}(E)$ as a von Neumann algebra, it follows that the map $R \mapsto \mathfrak{I}(R)$ is a monomorphism of $O(E)$ into the group

$$
\operatorname{Aut}_{E}(\mathfrak{R})=\{\phi \in \operatorname{Aut}(\mathfrak{R}): \phi(E) \subset E\} .
$$

It is easy to see that, for any $\phi$ in $\operatorname{Aut}_{E}(\mathfrak{H})$, the induced linear map on $E$ is indeed an orthogonal operator; in other words, $O(E)$ is canonically isomorphic to the group $\operatorname{Aut}_{E}(\mathfrak{I})$ of special automorphisms of $\mathfrak{A}$.

Let Inn $(\mathfrak{P})$ denote the group of inner automorphisms of $\mathfrak{Q}(E)$. It is a normal subgroup of Aut ( $\mathfrak{2})$; indeed, if $\phi^{\prime}=\operatorname{Ad}(U)$ for some unitary $U$ in $\mathfrak{A}(E)$, then $\phi \phi^{\prime} \phi^{-1}=\operatorname{Ad} \phi(U)$ for any $\phi$ in Aut (a). We shall denote by $G_{2}$ the inverse image of $\operatorname{Inn}_{E}(\mathfrak{P})=\operatorname{Inn}(\mathfrak{Q}) \cap \operatorname{Aut}_{E}(\mathfrak{H})$ under the map $R \mapsto \mathfrak{A}(R)$. It is clear that $\operatorname{Inn}_{E}(\mathfrak{R})$ is normal in $\operatorname{Aut}_{E}(\mathfrak{Q})$ and that $G_{2}$ is normal in $O(E)$.

Lemma 1. The principal automorphism $\alpha=\mathfrak{A}(-I)$ of $\mathfrak{A}(E)$ is outer, so that $G_{2}$ is a proper normal subgroup of $O(E)$.

Proof. Suppose, on the contrary, that $\alpha=\operatorname{Ad} U$ with $U$ unitary in $\mathfrak{Y}(E)$. Let $N$ be the positive integers and let $\left(e_{n}\right)_{n \in N}$ be an orthonormal basis in $E$. Let $V_{n}=-\operatorname{Ad} e_{n}$. Then $V_{n}$ is a symmetry (self-adjoint unitary) on $H_{0}$. So $V_{n}$ is reflection in its maximal invariant subspace $L_{n}$. For any finite subset $S=\left\{n_{1}, \ldots, n_{k}\right\} \subset N$ with $n_{1}<\ldots<n_{k}$, let $e_{S}=e_{n_{1}} \ldots e_{n_{k}}$. The elements $e_{S}$ form an orthonormal basis of $H_{0}$. The following equation is easy to check:

$$
V_{n} e_{S}=\left\{\begin{array}{l}
e_{S} \text { when }|S| \text { odd and } n \notin S, \text { or }|S| \text { even and } n \in S \\
-e_{S} \text { otherwise. }
\end{array}\right.
$$

It follows immediately that $\cap L_{n}=0$. But for all $n$ we have

$$
V_{n}(U)=-e_{n} U e_{n}=\alpha\left(e_{n}\right) U e_{n}=U e_{n} U^{*} U e_{n}=U
$$

so that $U \in \cap L_{n}$, a contradiction. This proof is based on an idea of Blattner [2; p. 273].

Our task is to identify $G_{2}$. As a first step, we quote a global algebraic fact: any proper normal subgroup of $O(E)$ is in $O^{\max }(E)_{\infty}$ (see Proposition 1R in [8]). So we have

LeMmA 2. $G_{2}$ is a subgroup of $O^{\max }(E)_{\infty}$.

Let $\Gamma$ be a separable locally compact group. Let $r_{1}$ be a faithful continuous representation of $\Gamma$ into some orthogonal group $O\left(E_{1}\right)$, with $E_{1}$ a real separable Hilbert space (for example, $r_{1}$ may be the left regular representation of $\Gamma$ on realvalued $L^{2}$-functions). Let $r$ be an infinite countable direct sum of copies of $r_{1}$; then $r$ is a representation of $\Gamma$ on a Hilbert space that we may identify with $E$. Since the direct sum is infinite, it is clear that $r(\gamma)$ lies in $O^{\max }(E)_{\infty}$ if and only if $r(\gamma)=1$, that is if and only if $\gamma$ is the identity in $\Gamma$. Following Blattner, we let $\rho$ be the composite 
map : $\Gamma \stackrel{r}{\rightarrow} O(E) \rightarrow \operatorname{Aut}_{E}(\mathfrak{2})$. We have proved the following result of Blattner [2; p. 674].

COROLlary 3. Let $\Gamma$ be a separable locally compact group. Then $\Gamma$ has a special continuous automorphic group representation $\rho$ such that $\rho(\gamma)$ is outer unless $\gamma$ is the identity in $\Gamma$.

By a special automorphic group representation we mean a homomorphism $\rho: \Gamma \rightarrow \operatorname{Aut}_{E}(\mathfrak{2})$; it is continuous if the map $\gamma \rightarrow \rho(\gamma) X$ of $\Gamma$ into $\mathfrak{A}(E)$ is continuous for each $X$ in $\mathfrak{A}(E)$, with the weak operator topology on $\mathfrak{A}(E)$. The special case of Corollary 3 when $\Gamma$ is a countable discrete group was obtained by Suzuki [24]. Other proofs are due to Choda [25] and to Nakamura and Takeda [26].

\section{Proofs of Theorems 1 and 2: first part}

We shall write $U(\mathscr{C})$ (respectively $U(\mathfrak{A})$ ) for the group of unitary elements in $\mathscr{C}(E)$ (respectively $\mathfrak{A}(E)$ ) furnished with the norm topology (respectively strong operator topology). It is clear the $U(\mathscr{C})$ is a complete metric group, but the case of $U(\mathfrak{R})$ necessitates a lemma (which could be traced to Lemma 1.3.2 in Murray and von Neumann [12]). We denote by $\mathfrak{A}(E)_{1}$ the unit ball $\left\{X \in \mathfrak{A}(E):\|X\|_{\infty} \leqslant 1\right\}$. Recall that $\mathfrak{U}(E)$ is identified with a subset of the Hilbert space $H_{0}$, with norm $\|\cdot\|_{2}$.

LEMMA 4. The topology of $U(\mathfrak{A})$ is that of a complete metric group with metric given by $d(U, V)=\|U-V\|_{2}$.

Proof. We denote in this proof by $\Omega$ rather than by 1 the trace vector in $H_{0}$ determined by the trace $\tau$. Then $\|X\|_{2}=\|X \Omega\|_{2}$ for all $X$ in $\mathfrak{A}(E)$. The vector $\Omega$ is cyclic and separating for $\mathfrak{A}(E)$, hence also for the commutant $\mathfrak{A}(E)^{\prime}$.

Let $\left(X_{\beta}\right)_{\beta \in B}$ be a net in $\mathfrak{A}(E)_{1}$ converging in the strong operator topology to some $X$ in $\mathfrak{U}(E)_{1}$. Then $\left(X_{\beta} \Omega\right)$ is a net in $H_{0}$ converging to $X \Omega$, so that $\left(X_{\beta}\right)$ converges to $X$ in the $\|\cdot\|_{2}$-topology.

Suppose conversely that $\left(X_{\beta}\right)$ is a net in $\mathfrak{U}(E)_{1}$ converging in the $\|\cdot\|_{2}$-topology to some $X$ in $\mathfrak{U}(E)_{1}$. For all $Y^{\prime}$ in $\mathfrak{A}(E)^{\prime}$ we have

$$
\begin{aligned}
\left\|X_{\beta} Y^{\prime} \Omega-X Y^{\prime} \Omega\right\|_{2}=\left\|Y^{\prime}\left(X_{\beta}-X\right) \Omega\right\|_{2} & \leqslant\left\|Y^{\prime}\right\|_{\infty} \cdot\left\|\left(X_{\beta}-X\right) \Omega\right\|_{2} \\
& =\left\|Y^{\prime}\right\|_{\infty}\left\|X_{\beta}-X\right\|_{2} .
\end{aligned}
$$

Hence the net $\left(X_{\beta}\right)$ in $H_{0}$ converges to $X \xi$ for any vector $\xi$ in $\mathfrak{2}(E)^{\prime} \Omega$; as the latter lies dense in $H_{0}$, this holds also for any $\xi$ in $H_{0}$, so that $\left(X_{\beta}\right)$ converges strongly to $X$.

We have shown that the strong operator topology and the $\|\cdot\|_{2}$-topology coincide on $\mathfrak{A}(E)_{1}$; they coincide a fortiori on $U(\mathfrak{U})$.

The equality $X Y-X_{0} Y_{0}=X\left(Y-Y_{0}\right)+\left(X-X_{0}\right) Y_{0}$ shows that multiplication is strongly continuous on $\mathfrak{P}(E)_{1}$. The involution $X \mapsto X^{*}$ is an isometry in the $\|\cdot\|_{2}$ norm, since

$$
\left\|X^{*}\right\|_{2}^{2}=\tau\left(X X^{*}\right)=\tau\left(X^{*} X\right)=\|X\|_{2}^{2}
$$

for all $X$ in $\mathfrak{A}(E)$. Hence $U(\mathfrak{U})$ with the strong operator topology is indeed a topological group. Finally, the metric group $U(\mathfrak{I})$ is complete because it is strongly closed in $\mathfrak{I}(E)_{1}$, which is strongly complete by von Neumann's density theorem. This ends the proof. 
Let $R \in S O(E)_{\infty}$. The spectral theorem for compact operators implies that there exists a decomposition into orthogonal planes $E=\bigoplus_{n \in N} E_{n}$ such that the restriction $R \mid E_{n}$ is a plane rotation $R\left(\theta_{n}\right)$ through an angle $\theta_{n} \in(-\pi, \pi]$ for each $n \in N$; as $R-I$ is compact, the sequence $\left(\theta_{n}\right)$ converges to 0 . It is then easy to compute that

$$
\begin{aligned}
& \|I-R\|_{1}=2^{3 / 2} \sum_{n \in N}\left(1-\cos \theta_{n}\right)^{1 / 2} \\
& \|I-R\|_{2}=2\left\{\sum_{n \in N}\left(1-\cos \theta_{n}\right)\right\}^{1 / 2}
\end{aligned}
$$

where $\|\cdot\|_{1}$ and $\|\cdot\|_{2}$ denote respectively the nuclear and the Hilbert-Schmidt norms of operators on $E$, namely

$$
\begin{aligned}
\|T\|_{1} & =\operatorname{Tr}|T|, \\
\|T\|_{2} & =\left\{\operatorname{Tr}|T|^{2}\right\}^{1 / 2}
\end{aligned}
$$

where $|T|=\left(T^{*} T\right)^{\frac{1}{2}}$. Since $1-\cos \theta \sim \theta^{2} / 2$ as $\theta \rightarrow 0$, it follows that

(i) $R \in S O(E)_{1}$ if and only if $\left(\theta_{n}\right)$ is in $l^{1}$

(ii) $R \in S O(E)_{2}$ if and only if $\left(\theta_{n}\right)$ is in $l^{2}$.

Let $\left(e_{n}\right)$ be an orthonormal basis in $E$ such that $\left\{e_{2 n-1}, e_{2 n}\right\}$ span $E_{n}$ for each $n$. Define

$$
\begin{aligned}
U_{n} & =e_{2 n-1}\left\{\cos \left(\theta_{n} / 2\right) e_{2 n-1}-\sin \left(\theta_{n} / 2\right) e_{2 n}\right\} \\
& =\cos \left(\theta_{n} / 2\right)-e_{2 n-1} e_{2 n} \sin \left(\theta_{n} / 2\right) \\
V_{n} & =U_{1} U_{2} \ldots U_{n}
\end{aligned}
$$

which are clearly all unitary and even elements in $\mathscr{C}(E)$, and a fortiori in $\mathfrak{A}(E)$.

Lemma 5. If $m, n \in N$ with $m<n$, then

(i) $\left\|V_{m}-V_{n}\right\|_{\infty}=2 \sin \left(\left|\theta_{m+1}\right|+\ldots+\left|\theta_{n}\right|\right) / 4$, provided that $\left|\theta_{m+1}\right|+\ldots+\left|\theta_{n}\right| \leqslant 2 \pi$;

(ii) $\left\|V_{m}-V_{n}\right\|_{2}^{2}=2-2 \cos \theta_{m+1} / 2 \ldots \cos \theta_{n} / 2$.

Proof. (i) In order to compute the $\mathrm{C}^{*}$-norm of $V_{m}-V_{n}$ we shall work in a Fock representation of $\mathscr{C}(E)$, which must be well adapted to the basis $\left(e_{n}\right)$, namely to $R$; this device is used by Shale and Stinespring [20; Section 3.2]. To be quite explicit, let $J$ be the orthogonal complex structure defined on $E$ by

$$
J e_{2 n-1}=e_{2 n}, \quad J e_{2 n}=-e_{2 n-1}
$$

for each $n \in N$. The complex Hilbert space $E_{J}$ defined by $E$ and $J$ has an orthonormal basis $\left(f_{n}\right)$ with $f_{n}=e_{2 n-1}$ for each $n \in N$ (the sets underlying $E$ and $E_{J}$ coincide).

Let $H_{J}$ be the Fock space defined by $J$, which is the Hilbert space completion of the exterior algebra $\Lambda\left(E_{J}\right)$ furnished with its standard hermitian form; the space $H_{J}$ has an orthonormal basis consisting of the vectors

$$
\eta_{S}=f_{n_{1}} \wedge f_{n_{2}} \wedge \ldots \wedge f_{n_{k}}
$$

where $k$ runs over $\{0\} \cup N$ and where $S=\left\{n_{1}, \ldots, n_{k}\right\}$ runs over strictly increasing sequences of positive integers. To each basis vector $e_{n}$ of $E$ is associated an operator $C_{n}$ defined on $H_{J}$ by

$$
C_{n}(\eta)=f_{n} \wedge \eta \quad \text { for all } \eta \text { in } H_{J}
$$


it is a so-called creator; its adjoint $C_{n}{ }^{*}$ is an annihilator; they satisfy the canonical anticommutation relations (CAR):

$$
\begin{array}{ll}
C_{n} C_{n}{ }^{*}+C_{n}{ }^{*} C_{n}=1 & n \in N \\
C_{n} C_{m}+C_{m} C_{n}=C_{n}{ }^{*} C_{m}{ }^{*}+C_{m}{ }^{*} C_{n}{ }^{*}=0 . &
\end{array}
$$

It follows from the universal property of Clifford algebras that the assignments

$$
\pi_{J}\left(e_{2 n-1}\right)=C_{n}+C_{n}{ }^{*} \quad \pi_{J}\left(e_{2 n}\right)=i\left(C_{n}-C_{n}{ }^{*}\right)
$$

for each $n \in N$ extend uniquely to a morphism of $\mathrm{C}^{*}$-algebras $\pi_{J}: \mathscr{C}(E) \rightarrow L\left(H_{J} ; H_{J}\right)$ which is the Fock representation defined by $J$; it has a cyclic vector $\Omega_{J}$ which is the unit in $\Lambda\left(E_{J}\right)=H_{J}$ and which is called the Fock vacuum. It is easy to check that

and that

$$
\begin{aligned}
& \pi_{J}\left(e_{2 n-1} e_{2 n}\right)=i\left(C_{n}{ }^{*} C_{n}-C_{n} C_{n}{ }^{*}\right) \quad n \in N \\
& \pi_{J}\left(U_{n}\right) \eta_{S}= \begin{cases}\exp \left(i \theta_{n} / 2\right) \eta_{S} & n \in S \\
\exp \left(-i \theta_{n} / 2\right) \eta_{S} & n \notin S\end{cases}
\end{aligned}
$$

$$
\pi_{J}\left(U_{m+1} U_{m+2} \ldots U_{n}\right) \eta_{S}=\exp \left\{i\left( \pm \theta_{m+1} \pm \ldots \pm \theta_{n}\right) / 2\right\} \eta_{S}
$$

where $\theta_{j}$ has a minus sign if and only if $j \notin S$. Hence

$$
\pi_{J}\left(V_{m}-V_{n}\right) \eta_{s}=\omega\left\{1-\exp i\left( \pm \theta_{m+1} \pm \ldots \pm \theta_{n}\right) / 2\right\} \eta_{s}
$$

where $\omega$ is a complex number of modulus 1 .

The situation now is that the $\eta_{S}$ constitute an orthonormal basis in $H_{J}$ of eigenvectors for the operator $\pi_{J}\left(V_{m}-V_{n}\right)$; the norm of this operator is consequently the maximum modulus of the corresponding eigenvalues, which is precisely

$$
2 \sin \left(\left|\theta_{m+1}\right|+\ldots+\left|\theta_{n}\right|\right) / 4 \text {. }
$$

But $\mathscr{C}(E)$ is simple, $\pi_{J}$ is isometric, so the $\mathrm{C}^{*}$-norm of $V_{m}-V_{n}$ is indeed given by (i).

Notice incidentally that the notations are consistent: if $a, b$ are vectors in $E$, then

$$
\left\langle\pi_{J}(a b) \Omega_{J} \mid \Omega_{J}\right\rangle=\langle a \mid b\rangle+i\langle J a \mid b\rangle=\langle a \mid b\rangle_{J}
$$

which is their scalar product in $H_{J}$, while

$$
\left\langle\pi_{0}(a b) \Omega \mid \Omega\right\rangle=\langle a \mid b\rangle
$$

which is their scalar product computed in $H_{0}$. Notice also that, if $E$ were finitedimensional, $\pi_{J}$ would be the spin representation of $\mathrm{Cl}(E)^{\mathbb{C}} \cong \mathscr{C}(E)$, and that the equivalence class of $\pi_{J}$ would not depend on $J$. When, on the contrary, $E$ is infinitedimensional, this class does depend on $J$ : see, for example [11].

(ii) The proof of the second equality reduces to a short computation:

$$
\begin{aligned}
\left\|V_{m}-V_{n}\right\|_{2}{ }^{2} & =\tau\left\{\left(V_{m}-V_{n}\right)^{*}\left(V_{m}-V_{n}\right)\right\} \\
& =\tau\left(1-V_{m}{ }^{*} V_{n}-V_{n}{ }^{*} V_{m}+1\right) \\
& =2-\tau\left(V_{m}{ }^{*} V_{n}+V_{n}{ }^{*} V_{m}\right) \\
& =2-\tau\left(U_{m+1} \ldots U_{n}+U_{n}{ }^{*} \ldots U_{m+1}^{*}\right) \\
& =2-2 \cos \theta_{m+1} / 2 \ldots \cos \theta_{n} / 2
\end{aligned}
$$


since $\tau$ annihilates all of $\mathscr{C}(E)$ except the one-dimensional subspace spanned by 1 .

The following lemma covers one half of Theorems 1(ii) and 2(ii).

LEMma 6. (i) If $R \in S O(E)_{1}$, then the infinite product $\Pi U_{n}$ is convergent in $U(\mathscr{C})$ in the $\mathrm{C}^{*}$-norm and implements $\mathscr{C}(R)$.

(ii) If $R \in S O(E)_{2}$, then the infinite product $\Pi U_{n}$ is convergent in $U(\mathfrak{H})$ in the $\|\cdot\|_{2}$ norm and implements $\mathfrak{U}(R)$.

Proof. (i) Let $R \in S O(E)_{1}$; then $\left(\theta_{n}\right)$ lies in $l^{1}$. Therefore $\left(V_{n}\right)$ is a Cauchy sequence in $U(\mathscr{C})$ in the $C^{*}$-norm by Lemma $5(\mathrm{i})$. But $U(\mathscr{C})$ is a complete metric group, so that $\left(V_{n}\right)$ converges in $\mathrm{C}^{*}$-norm to $V$, say. Then $V$ implements $\mathscr{C}(R)$ by a straightforward continuity argument.

(ii) Let $R \in S O(E)_{2}$; then the series with positive terms $\Sigma\left(1-\cos \theta_{n}\right)$ converges. For the proof, let $c_{n}$ denote $\cos \theta_{n}$; as $\theta_{n} \rightarrow 0$, there exists $m_{0}$ with $c_{n}>0$ as soon as $n \geqslant m_{0}$. The partial products $c_{m_{0}} \ldots c_{n}$ converge to some real number $d \neq 0$ (see for example Theorem 14.41 in [11]). If $m, n \in N$ with $m_{0}<m<n$, it means that

$$
c_{m} \ldots c_{n} \rightarrow 1 \quad \text { as } m, n \rightarrow \infty .
$$

By Lemma 5(ii), this implies that $\left(V_{n}\right)$ is a Cauchy sequence in $U(\mathfrak{2})$ in the $\|\cdot\|_{2^{-}}$ topology, which converges by Lemma 4 to $V$, say. Then $V$ implements $\mathfrak{A}(R)$ by continuity. This ends the proof.

The preceding lemma shows that naive judgment is confirmed and that

$$
\begin{aligned}
& \mathscr{C}\left\{R\left(\theta_{1}\right) \oplus R\left(\theta_{2}\right) \oplus \ldots\right\}=\operatorname{Ad}\left(U_{1} U_{2} \ldots\right) \\
& \mathfrak{A}\left\{R\left(\theta_{1}\right) \oplus R\left(\theta_{2}\right) \oplus \ldots\right\}=\operatorname{Ad}\left(U_{1} U_{2} \ldots\right)
\end{aligned}
$$

where the infinite product in the first displayed equation converges in the $\mathrm{C}^{*}$-norm, and that in the second converges in the strong operator topology. Recall that each $U_{n}$ is a product of the vectors $e_{2 n-1}$ and $e_{2 n}^{\prime}=\cos \left(\theta_{n} / 2\right) e_{2 n-1}-\sin \left(\theta_{n} / 2\right) e_{2 n}$ in $E_{n}$, which is a plane in $E$. The angle between $e_{2 n-1}$ and $e_{2 n}^{\prime}$ is $\theta_{n} / 2$ and we have

$$
\operatorname{Ad}\left(e_{2 n-1} e_{2 n}^{\prime}\right) Z=-e_{2 n-1}\left(-e_{2 n}^{\prime} Z e_{2 n}^{\prime}\right) e_{2 n-1}
$$

for each $Z$ in the appropriate algebra, so that $\operatorname{Ad} U_{n}$ acts as a product of two reflections.

In this sense, any operator in $S O(E)_{1}$ (respectively $S O(E)_{2}$ ) is a product of countably many reflections in subspaces of codimension 1 . This is a satisfactory generalization of the well-known fact that each element of $S O(n)$ is a product of an even number of reflections in hyperplanes of $R^{n}$.

\section{Second part of the proofs}

The next move (Lemma 8) is to prove what is essentially the converse of Lemma 6. The following easy technical lemma could be part of the proof of Lemma 8(i), but it seems clearer to present it beforehand: it is essentially Lemma 3 of [20].

Lemma 7. Let $H, K$ be complex Hilbert spaces, let $A$ be a unitary operator on $H$ and let $B$ be a unitary operator on $K$. Then, for any unitary operator $C$ on $H$, there exists $\lambda \in S^{1}$ (the complex numbers of modulus 1 ) with

$$
\left\|C \otimes 1_{K}-A \otimes B\right\| \geqslant\left\|\lambda A \otimes 1_{K}-A \otimes B\right\| .
$$


Proof. As $A$ and $B$ are unitary, one has

and similarly

$$
\begin{aligned}
\left\|C \otimes 1_{K}-A \otimes B\right\| & =\left\|\left(C \otimes 1_{K}-A \otimes B\right) A^{-1} \otimes B^{-1}\right\| \\
& =\left\|\left(C A^{-1}\right) \otimes B^{-1}-1_{H} \otimes 1_{K}\right\|
\end{aligned}
$$

$$
\left\|\lambda A \otimes 1_{K}-A \otimes B\right\|=\left\|1_{H} \otimes \lambda B^{-1}-1_{H} \otimes 1_{K}\right\|=\left\|\lambda B^{-1}-1_{K}\right\| .
$$

The lemma results from the following fact: if $U$ (respectively $V$ ) is a unitary operator on $H$ (respectively $K$ ) then there exists $\lambda \in S^{1}$ with $\left\|U \otimes V-1_{H} \otimes 1_{K}\right\| \geqslant\left\|\lambda V-1_{K}\right\|$.

Let us prove this fact. We denote by $\sigma(X)$ the spectrum of an operator $X$. If $\mu \in \sigma(U)$ and $v \in \sigma(V)$ then $\mu \nu$ is clearly in $\sigma(U \otimes V)$ (incidentally, the converse holds [3]). Now choose $\lambda \in \sigma(U)$ which minimizes $\left\|\lambda V-1_{K}\right\|$; then

$$
\begin{aligned}
\left\|U \otimes V-1_{H} \otimes 1_{K}\right\| & =\sup \{|\omega-1|: \omega \in \sigma(U \otimes V)\} \\
& \geqslant \sup \{|\lambda \nu-1|: v \in \sigma(V)\} \\
& =\|\lambda V-1\| .
\end{aligned}
$$

Note that the lemma is not correct with $\lambda=1$ (look, for example, at the cases for which $U=-1_{H}, V=-1_{K}$ and $-C=A=1_{H}, B=-1_{K}$ ).

LEMMA 8. (i) Let $R \in O(E)$ with $R \in S O(E)_{\infty}$ or $-R \in S O(E)_{\infty}$. If $\mathscr{C}(R)$ is inner, then $R \in S O(E)_{1}$.

Proof. We work again in a Fock representation $\pi_{J}$ with $J$ adapted as above to $R$. Let $E_{J}$ be $E$ made complex and let $R_{J}$ be the unitary operator on $E_{J}$ corresponding to $R$. It is easy to check (on the basis vectors $\eta_{S}$ ) that

$$
\pi_{J}(R e)=\Lambda\left(R_{J}\right) \pi_{J}(e) \Lambda\left(R_{J}\right)^{-1}
$$

for all $e$ in $E$ (see also the lemma in Section 3 of [13]). Since $\pi_{J}$ is irreducible, there is at most one unitary operator on $H_{J}$ which implements $\pi_{J}(\mathscr{C}(R))$, up to a scalar multiple of modulus 1 . Consequently, if $\mathscr{C}(R)=$ Ad $W$ with $W$ unitary in $\mathscr{C}(E)$, then we may assume that $\pi_{J}(W)=\Lambda\left(R_{J}\right)$.

For each $n \in N$, let $F_{n}$ be the complex subspace of $E_{J}$ spanned by $f_{1}, \ldots, f_{n}$. Then

$$
H_{J}=\Lambda\left(E_{J}\right)=\Lambda\left(F_{n}\right) \otimes \Lambda\left(F_{n}{ }^{\perp}\right) .
$$

Let $R_{n}, R_{n}{ }^{\prime}$ be the restriction to $F_{n}, F_{n}{ }^{\perp}$ of $R_{J}$. Then

$$
\Lambda\left(R_{J}\right)=\Lambda\left(R_{n}\right) \otimes \Lambda\left(R_{n}{ }^{\prime}\right) .
$$

The * subalgebra of $\mathscr{C}(E)$ generated by the union of the $F_{n}$ is dense in $\mathscr{C}(E)$. Hence there exists for each $n \in N$ an element $W_{n}$ in $\mathrm{Cl}\left(F_{n}\right)^{\mathbb{C}}$ such that the sequence $\left(W_{n}\right)$ converges to $W$; we may choose each $W_{n}$ unitary (if not, replace it by the unitary part in its polar decomposition). For each $n \in N$, the operator $\pi_{J}\left(W_{n}\right)$ is of the form $Y_{n} \otimes 1$ on $\Lambda\left(F_{n}\right) \otimes \Lambda\left(F_{n}{ }^{\perp}\right)$, and is arbitrarily near $\Lambda\left(R_{n}\right) \otimes \Lambda\left(R_{n}{ }^{\prime}\right)$ if $n$ is large enough. By Lemma 7, we can choose $W_{n}$ such that $Y_{n}=\alpha_{n} \Lambda\left(R_{n}\right)$ for some $\alpha_{n}$ in $S^{1}$. Now

$$
\left(Y_{n} \otimes 1\right) \Omega_{J}=\alpha_{n} \Omega_{J} \rightarrow \Lambda\left(R_{J}\right) \Omega_{J}=\Omega_{J}
$$

so that $\alpha_{n} \rightarrow 1$, and we may assume that each $\alpha_{n}$ is 1 to start with. We have shown that the hypothesis $\mathscr{C}(R)=\mathrm{Ad} W$ implies that the sequence $\Lambda\left(R_{n}\right) \otimes 1$ converges to $\Lambda\left(R_{j}\right)$ in the $C^{*}$-norm. 
With the notations introduced in the proof of Lemma 5(i), the $\eta_{s}$ are the elements of an orthonormal basis of $H_{J}$ with respect to which $\Lambda\left(R_{n}\right) \otimes 1$ is diagonal for each $n \in N$. It follows readily that

$$
\left\|\Lambda\left(R_{n}\right) \otimes 1-\Lambda\left(R_{m}\right) \otimes 1\right\|=2 \sin \left\{\left|\theta_{m+1}\right|+\ldots+\left|\theta_{n}\right|\right\} / 2
$$

as soon as $m<n$. As $\Lambda\left(R_{n}\right) \otimes 1$ is a Cauchy sequence of operators on $H_{J}$, the sequence of non-negative numbers $\left|\theta_{n}\right|$ is summable and $R \in S O(E)_{1}$. This ends the proof, due essentially to Shale and Stinespring [20; p. 322].

Let $R \in S O(E)_{1}$; it is easy to verify that, with $V_{n}$ as in Lemma $5(i)$ and $V$ as in Lemma 6(i),

$$
\begin{gathered}
\left(\operatorname{det}\left(R_{n}\right)\right)^{1 / 2} \pi_{J}\left(V_{n}\right)=\Lambda\left(R_{n}\right) \\
\left(\operatorname{det}\left(R_{J}\right)\right)^{1 / 2} \pi_{J}(V)=\Lambda\left(R_{J}\right) .
\end{gathered}
$$

In other words, $\mathscr{C}(R)$ is implemented both by $\pi_{J}{ }^{-1}\left(\Lambda\left(R_{J}\right)\right)$ and $V$, which differ by a scalar of modulus 1: this number is a square root of the determinant of $R_{J}$. It may be helpful to recall the definition of $\Lambda\left(R_{J}\right): \Lambda\left(R_{J}\right)$ is the unique unitary on $\Lambda\left(E_{J}\right)$ such that

$$
\Lambda\left(R_{J}\right) f_{n_{1}} \wedge \ldots \wedge f_{n_{k}}=R_{J} f_{n_{1}} \wedge \ldots \wedge R_{J} f_{n_{k}}
$$

whenever $n_{1}<\ldots<n_{k}$.

LEMMA 8. (ii) Let $R \in O(E)$ with $R \in S O(E)_{\infty}$ or $-R \in S O(E)_{\infty}$. If $\mathfrak{A}(R)$ is inner, then $R \in S O(E)_{2}$.

Proof. Let $\mathfrak{U}(R)=\operatorname{Ad} U$. Since the $e_{S}$ constitute an orthonormal basis of $H_{0}$, there exists an index $S$ with $\left\langle U \mid e_{S}\right\rangle \neq 0$. Let $V=e_{S}^{*} U$. Then

$$
\langle V \mid 1\rangle=\left\langle e_{S}^{*} U \mid 1\right\rangle=\left\langle U \mid e_{S}\right\rangle \neq 0
$$

so that in particular $V$ is even. Define $R^{\prime}$ by $R^{\prime} e=e_{S}^{*} e e_{S}$ for each $e$ in $E$. Then $R^{\prime} \in O^{t}(E)_{2}$ (indeed, either $R^{\prime}-I$ or $R^{\prime}+I$ is of finite rank, depending on whether $|S|$ is even or odd). Let $T=R^{\prime} R$. Then

$$
\mathfrak{U}(T)=\mathfrak{U}\left(R^{\prime}\right) \mathfrak{A}(R)=\operatorname{Ad} e_{S}^{*} . \operatorname{Ad} U=\operatorname{Ad} V .
$$

But $R \in S O(E)_{2}$ if and only if $T \in S O(E)_{2}$. Thus we may assume without loss of generality that $U$ is even and that $\langle U \mid 1\rangle \neq 0$.

Let $V_{n}: H_{0} \rightarrow H_{0}$ be given by $V_{n}(X)=\left(R e_{n}\right) X e_{n}$ and let $M_{n}=\left\{X \in H_{0}: V_{n}(X)=X\right\}$ be the invariant subspace of $V_{n}$ for each $n \in N$; define $M$ to be the intersection of the $M_{n}$. For each $n \in N$, let $P_{n}=\left(1+V_{n}\right) / 2$ be the projection on $M_{n}$ and let $Q_{n}=P_{1} \ldots P_{n}$. Now the $V_{n}$ mutually commute so the $P_{n}$ mutually commute; therefore $\left(Q_{n}\right)$ is a monotonically decreasing sequence of projections, and so is a strongly convergent sequence (see $[7 ;$ p. 255]); the limit is the projection $Q$ on $M$. Now $U$, regarded as a vector in $H_{0}$, has the property that

$$
V_{n}(U)=\left(R e_{n}\right) U e_{n}=\left(U e_{n} U^{*}\right) U e_{n}=U
$$

for each $n \in N$; hence $U$ lies in $M$ and $P \leqslant Q$, where $P$ is the projection on the onedimensional subspace of $H_{0}$ spanned by $U$. Therefore

$$
0<|\langle U \mid 1\rangle|^{2}=\langle P(1) \mid 1\rangle \leqslant\langle Q(1) \mid 1\rangle=\lim _{n}\left\langle Q_{n}(1) \mid 1\right\rangle .
$$


It is easy to verify that

$$
\left\langle Q_{2 n-1}(1) \mid 1\right\rangle=\left\langle Q_{2 n}(1) \mid 1\right\rangle=\cos ^{2} \theta_{1} / 2 \ldots \cos ^{2} \theta_{n} / 2
$$

Therefore

$$
0<\prod_{n \in N} \cos ^{2} \theta_{n} / 2 \leqslant 1
$$

By an elementary result concerning infinite products, this implies that $\Sigma \sin ^{2} \theta_{n} / 2$ is convergent. Since $\sin \theta \sim \theta$ as $\theta \rightarrow 0$, this implies that $\Sigma \theta_{n}{ }^{2}$ is convergent and so $R \in S O(E)_{2}$. The ideas in this proof are again due to Blattner [2; p. 671].

Let Aut $(\mathscr{C})$ be the group of * automorphisms of $\mathscr{C}(E)$; we may assume that they are uniformly continuous, though we need not [18; p. 162]. Let Inn $(\mathscr{C})$ be the normal subgroup of inner automorphisms. We may identify

with $O(E)$ and

$$
\operatorname{Aut}_{E}(\mathscr{C})=\{\phi \in \operatorname{Aut}(\mathscr{C}): \phi(E) \subset E\}
$$

$$
\operatorname{Inn}_{E}(\mathscr{C})=\operatorname{Aut}_{E}(\mathscr{C}) \cap \operatorname{Inn}(\mathscr{C})
$$

with a normal subgroup $G_{1}$ of $O(E)$.

We shall denote by $S G_{1}$ (respectively $S G_{2}$ ) the group of those $R \in O(E)$ for which the inner automorphism $\mathscr{C}(R)$ (respectively $\mathfrak{A}(R)$ ) is even. It is clear that $G_{1} \subset G_{2}$ and that $S G_{1} \subset S G_{2}$.

LEMMA 9. (i) $S G_{1}$ is of index two in $G_{1}$.

(ii) $S G_{2}$ is of index two in $G_{2}$.

Proof. (i) Let $t$ be a unit vector in $E$ and let $T \in O(E)$ be defined by

$$
T e=-e+2\langle e \mid t\rangle t
$$

for all $e$ in $E$; one has $T^{2}=I$ and $T \in O^{b}(E)_{1}$. Then $\mathscr{C}(T)=\operatorname{Ad} t$ and $T \in G_{1}$, $T \notin S G_{1}$. If $R$ is in $O(E)$ then $\mathscr{C}(R)$ is inner odd if and only if $\mathscr{C}(R T)$ is inner even; hence $S G_{1}$ is of index two in $G_{1}$. Notice also that $R$ is in $O^{b}(E)_{1}$ if and only if $R T$ is in $S O(E)_{1}$. The proof of (ii) is similar.

Proof of Theorem 1. Let $R \in G_{1}$. By Lemma 2, one has either $R$ or $-R$ in $O^{t}(E)_{\infty}=$ $S O(E)_{\infty} \cup O^{b}(E)_{\infty}$. If one of $R,-R$ is in $S O(E)_{\infty}$, then $R \in S O(E)_{1}$ by Lemma $8(\mathrm{i})$. If one of $R,-R$ is in $O^{b}(E)_{\infty}$, then $R \in O^{b}(E)_{1}$ by Lemmas 9 (i) and 8(i). Hence $G_{1} \subset O^{t}(E)_{1}$. The opposite inclusion follows from Lemmas 6(i) and 9(i).

By Lemma 6(i) one has $S O(E)_{1} \subset S G_{1}$. As $S O(E)_{1}$ is of index two in $O^{t}(E)_{1}$ and as $S G_{1}$ is of index two in $G_{1}$, this implies that $S O(E)_{1}=S G_{1}$.

Proof of Theorem 2. The above argument works with the help of Lemmas 6(ii), 8(ii) and 9(ii).

\section{Remarks}

(1). It follows from Theorems 1 and 2 that $S G_{1}$ contains the derived group of $S G_{2}$. Indeed, let $R_{j}=I+X_{j} \in S O(E)_{2}$, so that $X_{j}+X_{j}{ }^{*}+X_{j} X_{j}{ }^{*}=0(j=1,2)$; if $R=R_{1} R_{2} R_{1}^{-1} R_{2}^{-1}$, then $R-I$ is a sum of products of two and more HilbertSchmidt operators, so that $R-I$ is nuclear and $R \in O(E)_{1}$; a connectedness argument 
shows that $R \in S O(E)_{1}$. A simple direct proof of this, in terms of $\mathscr{C}(E)$ and $\mathfrak{A}(E)$, would probably shed light on both theorems.

(2). Stronger versions of Theorems 1 and 2 in terms of universal covering groups of $S O(E)_{1}$ and $S O(E)_{2}$ appear in [9] and [15].

(3). The group $G_{1}^{\text {rep }}$ of those $R$ in $O(E)$ for which $\pi \circ \mathscr{C}(R)$ and $\pi$ are unitarily equivalent for any representation $\pi$ of $\mathscr{C}(E)$ coincides with $G_{1}$, namely with $O^{t}(E)_{1}$. This is due to Slawny [23]. We conjecture that the group $G_{2}^{\text {rep }}$ of those $R$ in $O(E)$ for which $\pi \circ \mathfrak{A}(R)$ and $\pi$ are unitarily equivalent for any normal representation $\pi$ of $\mathfrak{A}(E)$ coincides with $G_{2}$. Indeed, if $O(E)$ is identified with $\operatorname{Aut}_{E}(\mathfrak{U})$, then $G_{2}^{\text {rep }}=$ $O(E) \cap N$ where $N=\{\alpha \in$ Aut $(\mathfrak{U}): \pi \circ \alpha$ and $\pi$ are equivalent for any normal

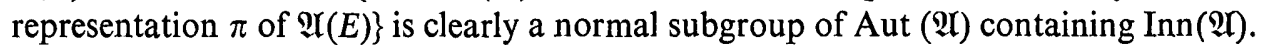
Now if one could check that $N \neq$ Aut ( $\mathfrak{A})$, it would follow from a result of Connes [5; Corollary 4] that $N=\operatorname{Inn}(\mathfrak{A})$. Consequently $G_{2}^{\text {rep }}=O(E) \cap \operatorname{Inn}(\mathfrak{A})$ would be equal to $G_{2}=O^{t}(E)_{2}$.

(4). The problem remains of characterizing those automorphisms of $\mathscr{C}(E)$ or $\mathfrak{A}(E)$ that can be written as $\mathscr{C}(R)$ or as $\mathfrak{U}(R)$ for some $R$ in $S O(E)_{\infty}$. Results in [16] and [22] make it hard to guess the answer.

\section{References}

1. M. F. Atiyah, R. Bott and A. Shapiro, "Clifford modules", Topology 3, supplement 1 (1964), 3-38.

2. R. J. Blattner, "Automorphic group representations ", Pacific J. Math., 8 (1958), 665-677.

3. A. Brown and C. Pearcy, "Spectra of tensor products of operators", Proc. Amer. Math. Soc., 17 (1966), 162-166.

4. J. C. Burkill and H. Burkill, Mathematical Analysis 2 (Cambridge University Press, 1970).

5. A. Connes, "Outer conjugacy classes of automorphisms of factors", Ann. Sci. Ecole. Norm. Sup., 8 (1975), 383-419.

6. J. Dixmier, Les algèbres d'opérateurs dans l'espace hilbertien (algèbres de von Neumann), 2nd edition (Gauthier-Villars, Paris, 1969).

7. P. R. Halmos, $A$ Hilbert space problem book (Springer-Verlag, Berlin, 1974).

8. P. de la Harpe, "Sous-groupes distingués du groupe unitaire et du groupe général linéaire d'un espace de Hilbert", Comment. Math. Helv., 51 (1976), 241-257.

9. P. de la Harpe, "The Clifford algebra and the spinor group of a Hilbert space ", Compositio Math., 25 (1972), 245-261.

10. N. M. Hugenholtz and R. V. Kadison, "Automorphisms and quasi-free states on the CAR algebra ", Comm. Math. Phys., 43 (1975), 181-197.

11. J. Manuceau and A. Verbeure, "The theorem on unitary equivalence of Fock representations ", Ann. Inst. H. Poincaré, 16 (1971), 87-91.

12. F. J. Murray and J. von Neumann, " On rings of operators IV ", Ann. Math., 44 (1943), 716-808.

13. R. J. Plymen, "Spinors in Hilbert space", Math. Proc. Cambridge Philos. Soc., 80 (1976), $337-347$.

14. R. J. Plymen, "Some recent results on infinite-dimensional spin groups", Advances in Math. supplementary studies 6 (1979), 159-171.

15. R. J. Plymen and R. F. Streater, "A model of the universal covering group of $S O(E)_{2}$ ", Bull. London Math. Soc., 7 (1975), 283-288.

16. R. J. Plymen and R. M. G. Young, "On the spin algebra of a real Hilbert space ", J. London Math. Soc., 9 (1974), 286-292.

17. C. R. Putnam and A. Winter, "The orthogonal group in Hilbert space ", Amer. J. Math., 74 (1952), 52-78.

18. S. Sakai, $C^{*}$-algebras and $W^{*}$-algebras (Springer, Berlin, 1971).

19. R. Schrader and D. A. Uhlenbrock, "Markov structures on Clifford algebras", J. Functional Analysis, 18 (1975), 389-413.

20. D. Shale and W. F. Stinespring, "Spinor representations of infinite orthogonal groups", J. Math. Mech., 14 (1965), 315-322. 
21. D. Shale and W. F. Stinespring, "States of the Clifford algebra ", Ann. Math., 80 (1964), 365-381.

22. R. F. Streater, "Interpolating norms for orthogonal and spin Lie algebras ", Symposia Math., 14 (1974), 173-179.

23. Y. Slawny, "Representations of canonical anti-commutation relations and implementability of canonical transformations ", Thesis, The Weizmann Institute of Science, Rehovot 1969.

24. N. Suzuki, "A linear representation of a countably infinite group ", Proc. Japan Acad., 34 (1958), 575-579.

25. H. Choda, "On a simple representation of groups as outer automorphism groups of the hyperfinite II $_{1}$ factor", Mem. Osaka Kyoiku Univ. III Natur. Sci. Appl. Sci., 20 (1971), 113-115.

26. M. Nakamura and Z. Takeda, "On certain examples of the crossed product of finite factors, II ", Proc. Japan Acad., 34 (1958), 500-502.

Section de Mathématiques,

Université de Genève, Case postale 124, 1211 Geneva 24,

Switzerland.
Department of Mathematics, The University, Manchester M13 9PL. 